\title{
AUTOMORPHISMS OF NILPOTENT-BY-ABELIAN GROUPS
}

\author{
C.K. Gupta and Frank Levin
}

\section{Dedicated to B.H. Neumann on the occasion of his eightieth birthday}

\begin{abstract}
For $F$ free of rank 4, a generating set for Aut $\left(F /\left[F^{\prime}, F^{\prime}, F^{\prime}\right]\right)$ exhibiting countably many wild automorphisms is obtained. Also included are examples of wild automorphisms in Aut $\left(F / V_{k+1}\right)$ which are tame in Aut $\left(F / V_{k}\right)$ for $k \geqslant 1$, where $V_{k}=\left[F^{\prime \prime}, F, \ldots, F\right](F$ repeals $k$ times).
\end{abstract}

\section{IN'TRODUCTION}

A classical result of Nielsen [9] implies that all automorphisms of free abelian groups of finite ranks are tame, that is, induced by automorphisms of free groups. The analogous result holds for free metabelian groups of rank 2 (Bachmuth [1]) and for any finite rank different from 3 (Bachmuth and Mochizuki [3]), being false for rank 3 (Chein [7], Bachmuth and Mochizuki [2, 4]). Recently, Stöhr [10] has shown that the automorphisin group of a free centre-by-metabelian group of finite rank at least 4 is generated by the tame automorphisms and at most one additional automorphism. In this paper we consider extensions of the above results to the free groups in the variety of nilpotent of class 2-by-abelian groups, $F /\left[F^{\prime}, F^{\prime}, F^{\prime}\right]$. For $F$ free of rank 4 we obtain a generating set for Aut $\left(F /\left[F^{\prime \prime}, F^{\prime}, F^{\prime}\right]\right)$ consisting of tame automorphisms and countably many wild, that is non-tame, automorphisms. For $F / V_{k+1}, k \geqslant 1$, where $V_{k+1}=\left[F^{\prime \prime}, F, \ldots, F\right](k+1$ times $)$, we give examples of wild automorphisms of $F / V_{k+1}$ which are trivial on $F / V_{k}, k \geqslant 1$.

\section{Class-2-By-abelian variety}

For $F$ free of rank 2 the results of Stöh $[10]$ imply that Aut $\left(F /\left[F^{\prime}, F^{\prime}, F^{\prime}\right]\right)$ and Aut $\left(F / V_{k}\right), k \geqslant 1$, are infinitely-generated. By Bachnuth and Mochizuki [3], this is also true for $F$ of rank 3 . Hence, in the sequel we shall restrict our attention to the case where $F$ has rank at least 4 . Each of the varieties to be considered will contain the variety of metabelian groups and will have relatively free groups whose derived groups are nilpotent. For such varieties results of Bachmuth and Mochizuki [4] together with a

Received 2 May 1989

The research of the first author was supported by NSERC Canada.

Copyright Clearance Centre, Inc. Serial-fee code: 0004-9729/89 \$A2.00+0.00. 
result of Bryant and C.K. Gupta [6] imply that for any rank exceeding 3 the respective automorphism groups are generated by the tame automorphisms together with those which are trivial modulo $F^{\prime \prime}$, so in the sequel we may restrict our attention mainly to automorphisms of the latter type.

Let $G=G_{4}=F /\left[F^{\prime}, F^{\prime}, F^{\prime}\right]$ be the free class-two-by-abelian group of rank four freely generated by $x, y, u, v$. Let $A$ be the subgroup of Aut $(G)$ consisting of all those $I A$-automorphisms of $G$ which are trivial modulo $G^{\prime \prime}$ and let $T$ denote the group of tame automorphisms of $G$. Then it is clear that $A$ is the normal closure under $T$ of all automorphisms of the form $x \rightarrow x\left[\left[z_{1}, z_{2}\right]^{g},\left[z_{3}, z_{4}\right]^{k}\right], y \rightarrow y, u \rightarrow u, v \rightarrow v$, where $z_{i} \in\{x, y, u, v\}$ and $g, h \in G$. With $g \equiv x^{a} y^{b} u^{c} v^{d}\left(\bmod G^{\prime}\right), h \equiv x^{a^{\prime}} y^{b^{\prime}} u^{c^{\prime}} v^{d^{\prime}}\left(\bmod G^{\prime}\right)$, $a, b, c, d, a^{\prime}, b^{\prime}, c^{\prime}, d^{\prime} \in Z$, we define automorphisms $\alpha[g ; h], \beta[g ; h], \gamma[g ; h], \delta[g ; h]$ and $\varepsilon[g ; h]$ of $G$ as follows:

$$
\begin{aligned}
& \alpha[g ; h]: x \rightarrow x\left[[x, y]^{g},[u, v]^{h}\right], y \rightarrow y, u \rightarrow u, v \rightarrow v \\
& \beta[g ; h]: x \rightarrow x\left[[x, y]^{g},[y, v]^{h}\right], y \rightarrow y, u \rightarrow u, v \rightarrow v \\
& \gamma[g ; h]: x \rightarrow x\left[[x, y]^{g},[x, v]^{h}\right], y \rightarrow y, u \rightarrow u, v \rightarrow v \\
& \delta[g ; h]: x \rightarrow x\left[[x, v]^{g},[x, v]^{h}\right], y \rightarrow y, u \rightarrow u, v \rightarrow v \\
& \varepsilon[g ; h]: x \rightarrow x\left[[u, y]^{g},[u, v]^{h}\right], y \rightarrow y, u \rightarrow u, v \rightarrow v .
\end{aligned}
$$

Further, with $x_{1}=x, x_{2}=y, x_{3}=u$ and $x_{4}=v$, we define tame automorphisms $\tau\left[x_{i} \leftrightarrow x_{j}\right], \tau\left[x_{i} \rightarrow x_{i} x_{j}^{a}\right], \tau\left[x_{i} \rightarrow x_{j}^{a} x_{i}\right], a \in \mathbb{Z}$, and $\tau\left[x_{i} \rightarrow x_{i}^{-1}\right]$ of $G$ as follows:

$$
\begin{aligned}
& \tau\left[x_{i} \leftrightarrow x_{j}\right]: x_{i} \rightarrow x_{j}, x_{j} \rightarrow x_{i}, x_{k} \rightarrow x_{k} \text { for } k \neq i, j ; \\
& \tau\left[x_{i} \rightarrow x_{i} x_{j}^{a}\right]: x_{i} \rightarrow x_{i} x_{j}^{a}, x_{k} \rightarrow x_{k} \text { for } k \neq i ; \\
& \tau\left[x_{i} \rightarrow x_{j}^{a} x_{i}\right]: x_{i} \rightarrow x_{j}^{a} x_{i}, x_{k} \rightarrow x_{k} \text { for } k \neq i ; \\
& \tau\left[x_{i} \rightarrow x_{i}^{-1}\right]: x_{i} \rightarrow x_{i}^{-1}, x_{k} \rightarrow x_{k} \text { for } k \neq i .
\end{aligned}
$$

In particular, $\tau\left[x_{i} \rightarrow x_{j}^{-a} x_{i} x_{j}^{a}\right]=\tau\left[x_{i} \rightarrow x_{j}^{-a} x_{i}\right] \tau\left[x_{i} \rightarrow x_{i} x_{j}^{a}\right]$.

We begin with a technical lemma, the proof of which will further serve to illustrate the techniques involved in the subsequent arguments.

LEMMA 1.

(i) For $\rho=r[y \rightarrow y x]$ the conjugate $\alpha\left[x^{a} ; 1\right]^{\rho}=\left(\alpha\left[x^{a} ; 1\right]\right)^{\rho}$, $a \in Z$, is given by

$$
\alpha\left[x^{a} ; 1\right]^{p}=\left(\alpha\left[x^{a+1} ; 1\right]\right)\left(\alpha\left[y^{a} ; 1\right]\right)^{\nu \sigma},
$$

where $\sigma=\tau[x \leftrightarrow y]$ and $\nu=\tau\left[u \leftrightarrow y u y^{-1}\right] \tau\left[v \leftrightarrow y v y^{-1}\right]$.

(ii) For $\rho=\tau\left[y \rightarrow y x^{-1}\right]$, the conjugate $\alpha\left[x^{a} ; 1\right]^{\rho}$ is given by

$$
\alpha\left[x^{a} ; 1\right]^{\rho}=\left(\alpha\left[x^{a-1} ; 1\right]\right)\left(\alpha\left[y^{a-1} ; 1\right]\right)^{\sigma},
$$

where $\sigma=\tau[x \leftrightarrow y]$. 
Proof: By definition we have

$$
\begin{aligned}
& x \alpha\left[x^{a} ; 1\right]^{\rho}=x \rho^{-1} \alpha\left[x^{a} ; 1\right] \rho=x \alpha\left[x^{a} ; 1\right] \rho=\left(x\left[[x, y]^{x^{a}},[u, v]\right]\right) \rho \\
& =x\left[[x, y x]^{x^{a}},[u, v]\right]=x \alpha\left[x^{a+1} ; 1\right] \\
& y \alpha\left[x^{a} ; 1\right]^{\rho}=\left(y x^{-1}\right) \alpha\left[x^{a} ; 1\right] \rho=\left(y\left[[x, y]^{x^{a}},[u, v]\right]^{-1} x^{-1}\right) \rho=y x\left[[x, y x]^{x^{a}},[u, v]\right]^{-1} x^{-1} \\
& =y\left[[x, y]^{x^{a}},[u, v]^{x^{-1}}\right]^{-1}=\left(x\left[[x, y]^{y^{a}},[u, v]^{y^{-1}}\right]\right)^{\sigma} \\
& \quad=\left(x\left[[x, y]^{y^{a}},\left[u^{y^{-1}}, v^{y^{-1}}\right]\right]\right)^{\sigma}=\left(x\left[[x, y]^{y^{a}},[u, v]\right]\right)^{\nu \sigma}=y\left(\alpha\left[y^{a} ; 1\right]\right)^{\nu \sigma}, \\
& u \alpha\left[x^{a} ; 1\right]^{\rho}=u=u\left(\alpha\left[x^{a+1} ; 1\right]\right)\left(\alpha\left[y^{a} ; 1\right]\right)^{\nu \sigma} \\
& v \alpha\left[x^{a} ; 1\right]^{\rho}=v=v\left(\alpha\left[x^{a+1} ; 1\right]\right)\left(\alpha\left[y^{a} ; 1\right]\right)^{\nu \sigma}
\end{aligned}
$$

This completes the proof of (i)

The proof for (ii) is analogous.

The following lemma serves to reduce the generating set of Aut $(G)$ to some extent.

Lemma 2. $A=\langle\alpha[g ; h] \mid g, h \in G\rangle^{T}$.

Proof: It is straightforward to verify that

(i) $\beta[g ; h]=\alpha\left[g \rho^{-1} ; h \rho^{-1}\right]^{\rho}(\alpha[g ; y h])^{-1}$, where $\rho=\tau[u \rightarrow u y]$;

(ii) $\varepsilon[x g ; h]=\alpha\left[g \rho^{-1} ; h \rho^{-1}\right]^{\rho}(\alpha[g ; h])^{-1}$, where $\rho=\tau[x \rightarrow u x]$;

(iii) $\gamma[g ; h]=\alpha\left[g \rho^{-1} ; h \rho^{-1}\right]^{\rho}\left(\alpha\left[h_{1} ; g_{1} u^{-1}\right]\right)^{-\sigma}\left(\alpha\left[h_{1} u^{-1} ; g_{1} u^{-1}\right]\right)^{-\sigma}$, where $\rho=\tau[u \rightarrow u x], \sigma=\tau[x \leftrightarrow u] \tau[y \leftrightarrow v], g_{1}=g \sigma$ and $h_{1}=h \sigma$.

(iv) $\delta[g ; h]=\left(\gamma\left[g \rho^{-1} ; h \rho^{-1}\right]\right)^{\rho}(\gamma[v g ; h])^{-1}$, where $\rho=\tau[y \rightarrow y v]$;

and the proof fullows.

Lemma 3. Let $A_{1}=\left\langle\alpha\left[x^{m} ; 1\right] \mid m \geqslant 0\right\rangle^{T}$. Then we have

(i) $A_{1}=\left\langle\alpha\left[y^{m} ; 1\right] \mid m \geqslant 0\right\rangle^{T}$;

(ii) $\alpha\left[y^{-a} ; 1\right], \alpha\left[x^{-a} ; 1\right] \in A_{1}$ for all $a \geqslant 0$.

Proof: Using (4), $\alpha\left[x^{m+1} ; 1\right]^{\rho}=\alpha\left[x^{m} ; 1\right]\left(\alpha\left[y^{m} ; 1\right]\right)^{\sigma}$, where $\rho=\tau\left[y \rightarrow y x^{-1}\right]$ and $\sigma=\tau[x \leftrightarrow y]$, so it follows that for any $m \geqslant 0, \alpha\left[y^{m} ; 1\right] \in A_{1}$. The remainder of the argument is by induction on $m$, starting with the observation that $\alpha\left[y^{m} ; 1\right]=\alpha\left[x^{m} ; 1\right]$ for $m=0$. This proves (i).

For the proof of (ii), the fact that $\alpha\left[y^{-a}, 1\right] \in A_{1}$ follows from the observation that $\alpha\left[y^{-a} ; 1\right]=\left(\alpha\left[y^{a-1} ; 1\right]\right)^{-\sigma}$, where $\sigma=\tau\left[y \leftrightarrow y^{-1}\right]$. Using (3) and an induction on $a \geqslant 1$ finally yields $\alpha\left[x^{-a} ; 1\right] \in A_{1}$.

Corollary. $A_{1}=\left(\tau_{m} \mid m \geqslant 1\right)^{T}$, where $\tau_{m}$ is the automorphism given by

$$
\tau_{m}: x \rightarrow x\left[x, y ; u, v ; y^{m}\right], y \rightarrow y, u \rightarrow u, v \rightarrow v
$$


Proof: Observe that $\tau_{m}=\alpha[1 ; 1]^{-1} \alpha\left[y^{m} ; 1\right]^{\sigma}$, where $\sigma$ is the tame automorphism which conjugates $u$ and $v$ by $y^{m}$ and fixes the remaining generators.

\section{LEMMA 4.}

(i) For any $a, b \in \mathbf{Z}, \alpha\left[x^{a} y^{b} ; 1\right], \beta\left[x^{a} y^{b} ; 1\right] \in A_{1}$.

(ii) For any $a, b, c, d \in Z$ and $q \in g p\langle y, u, v\rangle, \alpha\left[x^{a} y^{b} u^{c} v^{d} ; q\right] \in A_{1}$.

Proof: (i) By Lemma 2(i), $\beta\left[x^{a} y^{b} ; 1\right]$ is in the normal closure of $\alpha\left[x^{a} y^{b} ; 1\right]$ and $\alpha\left[x^{a} y^{b} ; y\right]$, and since $\alpha\left[x^{a} y^{b} ; y\right]=\left(\alpha\left[x^{a} y^{b} ; 1\right]\right)^{\varphi}, \varphi=\tau\left[u \rightarrow u^{y}\right] \tau\left[v \rightarrow v^{y}\right]$, it suffices to show that $\alpha\left[x^{a} y^{b} ; 1\right] \in A_{1}$. Set $\alpha=\alpha\left[x^{a} y^{b} ; 1\right]$. The proof is by induction on the length of $|a|+|b|$. If either $a$ or $b$ is 0 , the proof follows from Lenma 3 . If $b<0$, then $\alpha^{\rho}=\left(\alpha\left[x^{a} y^{-b-1} ; 1\right]\right)^{-1}$, where $\rho=\tau\left[y \rightarrow y^{-1}\right]$. Hence, by induction, we may assume that $b>0$. If $b \geqslant|a|$, then conjugating $\alpha$ by $\sigma=\tau[x \rightarrow y x]$ if $a<0$ or by $\sigma=\tau\left[x \rightarrow y^{-1} x\right]$ if $a>0$ yields $a^{\sigma}=\alpha\left[x^{a} y^{b-|a|} ; 1\right]$. Hence we may assume further that $b<|a|$. If $\left.a>0, a^{\rho}=\alpha\left[x^{a-b-1} y^{b} ; 1\right]\left(\alpha \mid x^{b} y^{a-b-1} ; 1\right]\right)^{\sigma}$, where $\rho=\tau\left(y \rightarrow y x^{-1}\right)$, $\sigma=\tau[x \leftrightarrow y]$. If $a<0$, as in (3), $\alpha^{\rho}=\alpha\left[x^{a+b+1} y^{b} ; 1\right]\left(\alpha\left[x^{b} y^{a+b} ; 1\right]\right)^{-\nu \sigma}$, where $\rho=\tau[y \rightarrow y x], \sigma=\tau[x \leftrightarrow y]$ and $\nu=\tau\left[u \leftrightarrow y u y^{-1}\right] \tau\left[v \leftrightarrow y v y^{-1}\right]$. In either event, since $b<|a|$, this completes the inductive step.

(ii) Let $a, b, c, d$ be arbitrary, $q=1$ and set $\alpha_{1}=\alpha\left[x^{a} y^{b} u^{c} v^{d} ; 1\right]$. The proof is by induction on $|a|+|b|+|c|+|d|$. Conjugating $\alpha_{1}$ by $\tau\left[u \rightarrow v^{-1} u\right]$ if $c d>0$ or by $\tau[u \rightarrow v u]$ if $c d<0$, will lower $|a|+|b|+|c|+|d|$. Further, the proof for (i) carries over to give a reduction if both $a, b$ are non-zero. Hence, we nay assume that one of $c, d$ say $d$ is zero and one of $a, b$ is zero. If $b=0, a \in Z$ and $c>0$, then with $\rho=\tau\left[y \rightarrow y u^{-1}\right]$, $\sigma=\tau[y \leftrightarrow u], \alpha_{1}^{\rho}=\alpha\left[x^{a} u^{b-1} ; 1\right]\left(\beta\left[x^{a} y^{b-1} ; 1\right]\right)^{-\sigma} \in A_{1}$, by the induclion hypothesis and (i). If $c<0$, the analogous result is obtained with $\rho=\tau[y \rightarrow y u]$. Hence we may assume that $b \neq 0$. On the other hand, if $b \neq 0$ and $a=0$, then with $\rho=\tau\left[y \rightarrow y x^{-1}\right]$, $\sigma=\tau[x \leftrightarrow y],\left(\alpha\left[x^{b+1} u^{c} ; 1\right]\right)^{\rho}=\alpha\left[x^{b} u^{c} ; 1\right]\left(\alpha\left[y^{b} u^{c} ; 1\right]\right)^{\sigma}$, as in (4). Hence $\alpha_{1} \in A_{1}$. The proof of (ii) now by noting follows that for any $p \in G, q=y^{k} u^{m} v^{n}$, we have $(\alpha[p ; 1])^{\sigma}=$ $\alpha[p ; q]$, where $\sigma=\tau\left[u \rightarrow y^{-k} u y^{k}\right] \tau\left[v \rightarrow y^{-k} v y^{k}\right] \tau\left[u \rightarrow v^{-n} u v^{n}\right] \tau\left[v \rightarrow u^{-m} v u^{m}\right]$.

We now complete the proof of the following theorem.

THeOREM 1. Let $A$ be the subgroup of Aut $(G)$ consisting of all $I A$-automorphisms of $G$ which are trivial modulo $G^{\prime \prime}$ and let $A_{1}=\left\langle\alpha\left[x^{m} ; 1\right] \mid m \geqslant 0\right\rangle^{T}$, where $\alpha\left[x^{m} ; 1\right]$ is: $x \rightarrow x\left[[x, y]^{x^{m}},[u, v]\right], y \rightarrow y, u \rightarrow u, v \rightarrow v$. Then $A=A_{1}$.

Proof: To prove the theorem it suffices, by Lemma 2, to show that for any $p, q \in G, \alpha[p ; q] \in A_{1}$. Since $\alpha\left[p ; x^{a} y^{b} u^{c} v^{d}\right]=\left(\alpha\left[p^{\prime} ; x^{a}\right]\right)^{\sigma}$, where $\sigma=\tau[u \rightarrow$ $\left.y^{-b} u y^{b}\right] \tau\left[v \rightarrow y^{-b} v y^{b}\right] \tau\left[u \rightarrow v^{-d} u v^{d}\right] \tau\left[v \rightarrow u^{-c} v u^{c}\right]$, it suffices to assume that $q$ is a power of $x$. Conjugating $\alpha[1 ; 1]$ by $\rho=\tau\left[x \rightarrow x^{-1}\right] \tau[y \rightarrow y x]$ results in $(\alpha[1 ; 1])^{\rho}=$ $\alpha[x ; x](\alpha[1,1])^{\sigma}$, where $\sigma=\tau[x \leftrightarrow y]$, and hence $\alpha[x ; x] \in B$. More generally, 
$\alpha\left[x y^{b} u^{c} v^{d}, x y^{b} u^{c} v^{d}\right]=(\alpha[x, x])^{\rho} \in A_{1}$, where $\rho=\tau\left[x \rightarrow y^{b} x\right] \tau\left[x \rightarrow u^{c} x\right] \tau\left[x \rightarrow v^{d} x\right]$. On the other hand, if $\left(\alpha\left[x^{a} y^{b}, 1\right]\right)^{\sigma}=\alpha^{\prime}$, where $\sigma=\tau\left[u \rightarrow x^{-c} u x^{c}\right] \tau\left[v \rightarrow x^{-c} v x^{c}\right]$, then $x \alpha^{\prime}=x \alpha\left[x^{a} y^{b} ; x^{c}\right], y \alpha^{\prime}=y$,

$$
\begin{aligned}
u \alpha^{\prime} & =\left(\left(x^{c} u x^{-c}\right)\right) \alpha\left[x^{a} y^{b} ; 1\right] \sigma \\
& =\left(\left(x \alpha\left[x^{a} y^{b} ; x^{c}\right]\right)^{c} x^{-c}\right) u\left(x^{c}\left(x \alpha\left[x^{a} y^{b}, x^{c}\right]\right)^{-c}\right) \\
& =\left\{\prod_{m}\left(\left[[x, y]^{s},[u, v]^{t}\right]\right)\right\} u\left\{\prod_{m}\left(\left[[x, y]^{s},[u, v]^{t}\right]\right)^{-1}\right\} \\
& =u\left\{\prod_{m}\left(\left[[x, y]^{s u},[u, v]^{t u}\right]\right)\right\}\left\{\prod_{m}\left(\left[[x, y]^{s},[u, v]^{t}\right]\right)^{-1}\right\}
\end{aligned}
$$

where $s=x^{a} y^{b} x^{-m}, t=x^{c} y^{d} x^{-m}, m=1, \ldots, c$.

Hence

$$
u \alpha^{\prime}=u\left\{\prod_{m} \alpha\left[u^{c} v^{d} u^{-m} x, u^{a} v^{b} u^{-m} x\right]^{-\sigma}\right\}\left\{\prod_{m} \alpha\left[u^{c} v^{d} u^{-m}, u^{a} v^{b} u^{-m}\right]^{\sigma}\right\}
$$

where $\sigma=\tau[x \leftrightarrow u] \tau[y \leftrightarrow v]$ and, similarly,

$$
v \alpha^{\prime}=v\left\{\prod_{m}\left(\left[[x, y]^{n v},[u, v]^{t v}\right]\right)\right\}\left\{\prod_{n}\left(\left[[x, y]^{s},[u, v]^{t}\right)^{-1}\right\} .\right.
$$

It follows that $\alpha\left[x^{a} y^{b}, x^{c}\right] \in A_{1}$, for any $a, b, c \in Z$. The prool for general $p=x^{a} y^{b} u^{c} v^{d}$ now is completely analogous to that for Lemma 4(ii). This completes the proof.

To show that $\alpha\left[x^{n}, 1\right]$ is not induced by an automorphism of the free group $F$ of rank 4 with generators $x, y, u, v$, it suffices to show that for any choice of elements $w_{i} \epsilon$ $\left[F^{\prime}, F^{\prime}, F^{\prime}\right], i=1, \ldots, 4, F$ can nol be generated by $x[[x, y],[u, v]]\left[\left[x, y, x^{n}\right],[u, v] \mid w_{1}\right.$, $y w_{2}, u w_{3}, v w_{4}$. For this purpose the following general criterion due to Birman [5] is applicalble.

LeMma 5. (Birman[5]). Let $F_{n}$ be a free group of rank $n \geqslant 2$, freely generated by $x_{1}, \ldots, x_{n}$. An endomorplism $\phi$ of $F_{n}$ is surjective if and only if the $n \times n$ matrix $\left(\partial x_{i} \phi / \partial x_{j}\right)$ of the left Fox derivatives $\partial x_{i} \phi / \partial x_{j}$ of the $x_{i} \phi$ with respect to the $x_{j}$ is left-invertible over the free group ring $Z F_{n}$.

(For a discussion of Fox derivatives see, for instance, Narain Gupta [8].)

Let $\mathcal{M}_{4}$ denote the ring of $4 \times 4$ matrices over $Z F$ and $\phi$ be the endomorphism of $F$ defined by $x \phi=x\left[[x, y]\left[x, y, x^{n}\right] ;[u, v]\right] w_{1}, y \phi=y w_{2}, u \phi=u w_{3}, v \phi=v w_{4}$, for some $w_{i} \in\left[F^{\prime}, F^{\prime}, F^{\prime}\right]$. Let $M$ denote the matrix of the left Fox derivatives corresponding to $\phi$ as in Lemma 5 and $\psi$ a representation of $F$ in $G L_{2}(Z[z])$, over the polynomial ring $\mathrm{Z}[z]$ in the indeterminale $z$, defined by

$$
x \psi=\left[\begin{array}{cc}
1 & 0 \\
0 & 1
\end{array}\right], y \psi=\left[\begin{array}{cc}
1+z & -z \\
z & 1-z
\end{array}\right], u \psi=\left[\begin{array}{ll}
1 & z \\
0 & 1
\end{array}\right], v \psi=\left[\begin{array}{ll}
1 & 0 \\
z & 1
\end{array}\right]
$$


Then $\psi$ also defines a homomorphism of $Z F$ into $G L_{2}(Z[z])$ and, hence, a homomorphism of $\mathcal{M}_{4}$ into the ring of $8 \times 8$ (partitioned) matrices over $Z[z]$, which we may denote by $\psi$ also. For any $r \in \mathbf{f}^{k}$, the $k$-th power of the augmentation ideal $f$ of $Z F$, the entries of $r \psi$ will be in $z^{k} Z[z]$. In particular, the (Fox) derivative of any element in $\left[F^{\prime \prime}, F^{\prime}, F^{\prime}\right]$ will map to a matrix with entries in $z^{5} Z[z]$.

On the other hand,

$$
\begin{aligned}
(\partial x \phi / \partial x) \psi & =\left(1+([v, u]-1)\left(y^{-1}-1\right)\right) \psi \\
& =1 \psi+\left[\begin{array}{cc}
-z^{2} & -z^{3} \\
-z^{3} & z^{2}+z^{4}
\end{array}\right]\left[\begin{array}{cc}
z & -z \\
z & -z
\end{array}\right] \\
& =\left[\begin{array}{cc}
1-z^{3}-z^{4} & z^{3}+z^{4} \\
z^{3}-z^{4}+z^{5} & 1-z^{3}+z^{4}-z^{5}
\end{array}\right]
\end{aligned}
$$

and since the derivatives of $x \phi$ with respect to $y, u, v$ all have $(x-1)$ as a factor, they will map to the zero matrix. Thus it follows from a routine calculation modulo $z^{5}$, that $M \psi$ has a non-unit determinant in $\mathrm{Z}[z]$. Thus $M \psi$ is not invertible and, hence, $M$ is not left-invertible. We have thus proved the following result.

THEOREM 2. For all $n \geqslant 1$, the automorphism $\alpha\left[x^{n} ; 1\right]$ given by: $x \rightarrow x\left[[x, y]^{x^{n}},[u, v]\right], y \rightarrow y, u \rightarrow u, v \rightarrow v$, is not tame.

Finally, we ubserve that if the number of free generators of $F$ exceeds 4 , then $\alpha\left[x^{n} ; 1\right]$ can be extended by letting it act trivially on the additional generators and the above argument remains virtually unchanged, which means that Theorem 2 remains valid for $F$ of any finite rank $\geqslant 4$. We close this section with the conjecture that $\operatorname{Aut}\left(F /\left[F^{\prime}, F^{\prime}, F^{\prime}\right]\right)$ is not finitely generated for $F$ of rank 4 and, in particular, that the automorphisms $\alpha\left[x^{n}, 1\right], n \geqslant 0$, are independent modulo the tame automorphisms.

\section{AUTOMORPHISMS OF $\left(F / V_{k}\right)$}

In this section we exhibit, for each $k \geqslant 1$, a wild automorphism of $F / V_{k+1}$ which is trivial modulo $V_{k}$, where $F$ is free on $\left\{x, y, u, v, x_{1}, \ldots, x_{n}, n \geqslant 0\right\}$ and $V_{k}=\left[F^{\prime \prime}, F, \ldots, F\right]$ ( $F$ repeats $k$ times).

Theorem 3. Define $c_{1}=[[x, y],[u, v], v], c_{2}=[[x, y],[u, v], v, y]$ and, by induction, $c_{m}=\left[c_{m-1}, v\right]$ for odd $m, c_{m}=\left[c_{m-1}, y\right]$ for even $m$. If $\alpha_{k} \in \operatorname{Aut}\left(F / V_{k}\right)$ is defined by: $x \alpha_{k}=x c_{k-1}, y \alpha_{k}=y, u \alpha_{k}=u, v \alpha_{k}=v, x_{i} \alpha_{k}=x_{i}, i \geqslant 1$, then $\alpha_{k}$ is not tame.

Proof: As in the proof of Theorem 2, to show that $\alpha_{k}$ is not tame it suffices to show that the endomorphism $\phi$ of $F$, defined by $x \phi=x c_{k-1} w_{1}, y \phi=y w_{2}, u \phi=u w_{3}$, $v \phi=v w_{4}, x_{i} \phi=x_{i} w_{i+4}, i \geqslant 1$, is not surjective for any choice of $w_{i} \in V_{k}$, and 
this, in turn, is verified using the corresponding derivation matrix given by Lemma 5. For this purpose we define, as in the proof of Theorem 2, a representation $\psi$ of $F$ into $G L_{2}(Z[z])$ defined as in (5) on $x \phi, \ldots, v \phi$ and mapping the remaining $x_{i} \phi$ to the identity matrix. Since the $w_{i}$ are in $V_{k}$, their derivatives will be represented by matrices over $z^{k+4} Z[z]$, and modulo $z^{k+4}$ a routine computation shows that for the corresponding $(n+4) \times(n+4)$ matrix $M, M \psi$ is, as in the proof of Theorem 2, again a diagonal matrix with determinant different from \pm 1 , which completes the proof.

Concluding remarks. Let $G=F /\left[F^{\prime \prime}, F, F\right]$ be freely generated by $\{x, y, u, v, w\}$. Then since $\left[F^{\prime \prime}, F^{\prime \prime}\right]<\left[F^{\prime \prime}, F, F\right]$, it follows by Theorem 1 and the Corollary to Lemma 3 that, together with the tame automorphisms, Aut $(G)$ is generated by $\alpha[1 ; 1]$ and the automorphisms $\mu_{n}, n \geqslant 1$, defined by $\mu_{n}: x \rightarrow x\left[[x, y],[u, v], y^{n}\right], y \rightarrow y, u \rightarrow u$, $v \rightarrow v, w \rightarrow w$. Note that $\mu_{n}=\mu_{1}^{n}$. Taking $\tau$ to be the tame automorphism $x \rightarrow x$, $y \rightarrow y w, u \rightarrow u, v \rightarrow v, w \rightarrow w$ and $\kappa$ to be the tame automorphism conjugating $u$ and $v$ by $w$ and fixing the remaining generators gives $\mu_{1}=\alpha[1 ; 1]^{-1}(\alpha[1 ; 1])^{\tau \kappa}$. Thus, for rank $\geqslant 5, \operatorname{Aut}\left(F / V_{2}\right)$ is generated by the tame automorphisms together with a single non-tame automorphism $\alpha[1 ; 1]$. It seems that the same holds for Aut $\left(F / V_{k+1}\right)$ when the rank of $F$ is $\geqslant k+4$. We shall not go into details.

\section{REFERENCES}

[1] S. Bachmuth, 'Automorphisms of free metabelian groups', Trans. Amer. Math. Soc. 118 (1865), 93-104.

[2] S. Bachmull, and H.Y. Mochizuki, ' $I A$-Automorphisns of the free metabelian group of rank 3', J. Algebra 55 (1978), 106-115.

[3] S. Bachmuth and H.Y. Mochizuki, 'The nonfinite generation of Aut $(G), G$ free metabelian of rank 3', Trans. Amer. Meth. Soc. 270 (1982), 693-699.

[4] S. Bachmuth and H.Y. Mochizuki, 'Aut $(F) \rightarrow \operatorname{Aut}(F / F$ ') is surjective for free group $F$ of rank $\geqslant 4$ ', Trans. Amer. Math. Soc. 282 (1985), 81-101.

[5] J.S. Birman, 'An inverse function theorem for free groups', Proc. Amer. Math. Soc. 41 (1974), 634-638.

[6] R.M. Bryant and C.K. Gupta, 'Characteristic subgroups of free centre-by-metabelian groups', J. London Math. Soc.(2) 20 (1984), 435-440.

[7] O. Chein, ' $I$ A-automorphisms of free and free metabelian groups', Comm. Pure Appl. Math. 21 (1968), 605-629.

[8] Narain Gupta, Free Group Rings 66, Contemp. Matl. (Amer. Malh. Soc., 1987).

[8] J. Nielsen, 'Die Isomorphismengruppe der freien Gruppen', Math. Ann. 91 (1924), 169-209.

[10] E. Stöhr, 'On automorphisms of free centre-by-metabelian groups', Arch. Math. 48 (1987), 376-380.

Department of Mathematics

University of Manitoba

Winnipeg R3T' 2 N2

C'anada
Mathęmatisches Institut

Ruhr Universilät

463 Bochum

West Germany 